\title{
COMORBILIDAD ENTRE DISTRÉS PSICOLÓGICO Y ABUSO DE DROGAS EN PACIENTES DE CENTROS DE TRATAMIENTO: IMPLICACIONES EN POLÍTICAS Y PROGRAMAS, MANAGUA - NICARAGUA
}

\author{
Olga Vladimirovna Kulakova ${ }^{1}$, Robert Mann², Carol Strike ${ }^{3}$,Bruna Brands ${ }^{4}$, Akwatu Khenti ${ }^{5}$
}

\begin{abstract}
${ }^{1}$ MSc. Profesora Titular de la Universidad Nacional Autónoma de Nicaragua. León, Nicarágua. E-mail: olgvik20@yahoo.es
${ }^{2}$ Ph.D. Investigator. Centre for Addiction and Mental Health. University of Toronto. Toronto, Canada. E-mail: robert_mann@ camh.net

${ }^{3} \mathrm{Ph} . \mathrm{D}$. Investigator. Health Systems Research and Consulting Unit. Centre for Addiction and Mental Health. University of Toronto. Canada. E-mail: carol_strike@camh.net

${ }^{4}$ Ph.D. Investigator. Office of Research and Surveillance. Drug Strategy and Controlled Substances Programme. Health Canada and Public Health and Regulatory Policies. Center for Addiction and Mental Health. University of Toronto. Toronto, Canada. E-mail: bruna_brands@camh.net

${ }^{5}$ MSc. Director of Office of International Health Center for Addiction and Mental Health. University of Toronto. Toronto, Canada. E-mail: akwatu_khenti@camh.net
\end{abstract}

RESUMEN: El objetivo de este estudio fue determinar la prevalencia de la comorbilidad entre el distress psicológico y el abuso/ dependencia de drogas en los pacientes de los centros de tratamiento de Nicaragua. Se aplicaron cuestionarios EULAC-CICAD en forma de entrevista para describir el perfil del paciente y Kessler 10 para estimar niveles del distress a 130 usuarios de tres Centros de las Organizaciones no Gubernamentales de Managua, Nicaragua. Nivel encontrado de distress psicológico (severo y muy severo), fue de un $35 \%$. También se encontró que un número importante de los pacientes que están siendo tratados actualmente por problemas de salud mental, persisten en experimentar síntomas del distress psicológico. Se concluyó que los hallazgos de este estudio sugieren una revisión sistemática de las políticas y estrategias de intervención y el fortalecimiento de ambos sistemas involucrados, Sistema de Salud y de Drogodependencia.

DESCRIPTORES: Comorbilidad. Distrés psicológico. Trastornos relacionados con sustancias. Atención en salud mental.

\section{COMORBIDITY BETWEEN PSYCHOLOGICAL DISTRESS AND DRUG ABUSE TREATMENT CENTER PATIENTS: IMPLICATIONS FOR POLICIES AND PROGRAMS, MANAGUA - NICARAGUA}

\begin{abstract}
There is in patients with mental disorders a high frequency of psychopathology, by drug abuse. The simultaneous presence of both is Comorbidity and affects the quality of life of patients. The aim of this study was to determine the prevalence of comorbidity between psychological distress and abuse/dependence of drugs among patients in treatment centers in Nicaragua. The EULAC-CICAD and Kessler-10 was used for data collection in 130 patients over 18 years, in treatment by abuse/dependence on drugs in three Centers of Non-Governmental Organizations in Managua, Nicaragua. 35\% presented psychological distress. A significant number of patients currently being treated for mental health problems persist in experiencing symptoms of psychological distress. It was concluded that the findings of this study suggest a systematic review of policies and intervention strategies and the strengthening of both Systems of Health and Drug Dependency.
\end{abstract}

DESCRIPTORS: Comorbidity. Psychological distress. Substance use-related disorders. Mental health assistance.

\section{COMORBIDADE ENTRE SOFRIMENTO PSÍQUICO E ABUSO DE DROGAS EM PACIENTES DE CENTROS DE TRATAMENTO: IMPLICAÇÕES PARA AS POLÍTICAS E PROGRAMAS, MANAGUA - NICARAGUA}

RESUMO: O objetivo deste estudo foi determinar a prevalência da comorbidade entre sofrimento psíquico e o abuso/dependência de drogas nos pacientes ems centros de tratamento da Nicarágua. Foram utilizados questionários EULAC-CICAD na forma de entrevista para descrever o perfil dos pacientes e o Kessler 10 para estimar níveis de sofrimento psíquico em 130 usuários de três centros de Organizações não Governamentais de Manágua, Nicarágua. O nível encontrado de sofrimento psíquico (severo e muito severo) foi de $35 \%$. Foi verificado que um número importante de pacientes que estão sendo tratados atualmente para problemas de saúde mental, continuam apresentando sintomas desse distress psicológico. Conclui-se que os achados deste estudo sugerem una revisão sistemática das políticas e estratégias de intervenção e o fortalecimento dos dois sistemas envolvidos, o Sistema de Saúde e o de Dependências de Drogas. DESCRITORES: Comorbidade. Sofrimento psíquico. Transtornos relacionados ao uso de substancias. Assistência em saúde mental. 


\section{INTRODUCCIÓN}

La presencia simultánea (comorbilidad) de trastornos por uso de sustancias psicoactivas y otros trastornos mentales es frecuente y se traduce en un factor de riesgo para el tratamiento de pacientes que sufren estas patologías produciendo un mayor impacto en su calidad de vida y la de sus familias.

Las investigaciones dirigidas a aclarar los aspectos centrales de los trastornos mentales en las personas con adicciones han puesto en claro que la presencia simultánea de ambos problemas, produce déficit cognitivo, problemas interpersonales, daño emocional y biológico. ${ }^{1-3}$

Diferentes estudios estiman que aproximadamente el $70 \%$ de los pacientes con desordenes por consumo de sustancias, sufren comorbilidad con al menos un trastorno concomitante. ${ }^{4}$ Algunos estudios coinciden en evidenciar un aparente incremento de los casos de comorbilidad y otros sugieren una mejor contextualización del fenómeno con el fin de una mejor comprensión. La tasa de comorbilidad es elevada en los Centros de tratamiento para el abuso de drogas, porque las personas que tienen ambos problemas buscan el tratamiento más frecuentemente que aquellos que sólo tienen un problema. ${ }^{5}$

La comorbilidad es una realidad en los Centros de tratamiento por abuso/dependencia de drogas que suscita complejidad y un progresivo interés. Dicha complejidad puede provenir de la propia patología del paciente y los problemas internos que le genera (depresión, negación, alineación, etc.), pero de manera muy importante también puede derivarse de la descoordinación de diversos recursos asistenciales y los diferentes programas de tratamiento.

En la actualidad, el sistema de salud en Nicaragua enfrenta desafíos derivados de sus condiciones socioeconómicas. El presupuesto destinado a la salud mental es $1 \%$ del presupuesto general de la salud y, de este, $91 \%$ se destina al único hospital psiquiátrico ubicado en la capital del país, absorbiendo la gran mayoría de los recursos financieros consignados a la salud mental, en detrimento de los servicios especializados ambulatorios y de atención primaria. A esto se suma la falta de políticas nacionales de salud mental explicitas y la carencia de una legislación en esta materia. ${ }^{6}$

En materia de servicios de tratamiento por abuso/dependencia de sustancias psicoactivas, el Estado no cuenta con Centros especializados, las únicas entidades que albergan a los drogodependientes son Organizaciones No Gubernamentales
(ONGs) y privadas, que aún no están acreditadas por el Ministerio de Salud, por lo tanto la sociedad civil, a través de sus ONGs llevan mayor carga de intervención en esta problemática. La mayoría de los centros de tratamiento dirigen su atención a grupos en riesgo: niños, niñas y adolescentes. La oferta para la población adulta es muy limitada. ${ }^{6}$

En el campo investigativo en Nicaragua se reportan pocos datos relacionados con el tema; aporta alguna información a nivel nacional sobre la prevalencia de consumo de drogas, por ejemplo, un estudio realizado en la población general de 12 a 65 años demostró que entre 30,000 habitantes un $7.9 \%$ han consumido marihuana, $2.5 \%$ cocaína, $1.3 \%$ crack $^{7}$ y otro estudio, en la población escolar de 12 a 17 años, demostró que entre 5,412 escolares un $47.6 \%$ han consumido alcohol, $12.3 \%$ benzodiacepinas, 5.2\% marihuana, 2.3\% cocaína, $2 \%$ inhalantes y $1.2 \%$ crack $^{8}$ Entre los registros existentes sobre la prevalencia de salud mental, el trastorno bipolar se refleja como principal causa de asistencia psiquiátrica seguida por esquizofrenia. ${ }^{9}$

La falta de conocimiento informado sobre la realidad clínica de los pacientes que acuden a los centros de tratamiento por drogodependencia en Nicaragua, justificó la importancia de indagar más sobre esta población.

Se planteó el objetivo de determinar la prevalencia de la comorbilidad entre el distress psicológico y el abuso/dependencia de drogas en pacientes mayores de edad que acuden a los centros de atención por drogodependencia.

\section{MÉTODO}

En el período de febrero-marzo del 2010 en Managua, primer departamento de importancia en Nicaragua, se entrevistó a 130 usuarios mayores de 18 años que se encontraban en los centros de atención por abuso/dependencia de drogas. La muestra fue calculada en base de un $40 \%$ de prevalencia esperada del distress psicológico severo y muy severo de todo el universo elegible, de un total de 198 usuarios. Los Centros participantes fueron tres ONGs: "CENICSOL", ubicado en el pueblo Sabanas Grandes, "CARAS", en la ciudad de Managua y "REJESAD" en el municipio Tipitapa.

El diseño de estudio fue transversal usando como instrumentos de medición el formulario de admisión EULAC-CICAD, adaptado para este estudio con 30 preguntas sobre el estatus sociodemográfico, historia de abuso de drogas y alcohol y problemas mentales pasados y actuales y la escala 
Kessler-10 (K-10), una herramienta para evidenciar un posible problema de salud mental que requiera una mayor investigación en la población de adictos. ${ }^{10}$ Él cuestionario K-10 es de diez preguntas que mide el distress psicológico, el rango de los valores va desde 10 (indicando ausencia de distress psicológico) a 50 (distress severo). Distress psicológico se ha agrupado en cuatro niveles: bajo (10-15), moderado (16-21), severo (22-29) y muy severo (30-50). Para efectos analíticos, la mayoría de los estudios han unido las dos últimas categorías (distress severo y muy severo). Está demostrado que un nivel muy alto de distress psicológico obtenido a través de la puntuación de la escala K-10 está asociado con una alta probabilidad de tener un trastorno de ansiedad o depresión. ${ }^{11}$ En este estudio la fiabilidad del K-10 demostró una buena consistencia, con un valor Alpha de Cronbach 0.87 .

Todos los instrumentos se aplicaron en forma de entrevista, cara a cara. Para recolectar la información, se contó con la colaboración del Instituto Nacional Contra el Alcoholismo y Drogodependencia de Nicaragua (ICAD) y con los directores de los Centros de atención, que permitieron el acceso a los usuarios. Los participantes accedieron a la entrevista de manera voluntaria, firmando el consentimiento informado. Para el procesamiento y análisis estadístico de datos se utilizó el paquete informático SPSS, v.15.0.

El estudio fue aprobado por la Oficina de Ética en Investigación del Centro de Adicciones y Salud Mental de Canadá (CAMH) y por el Comité de Ética de Investigaciones Biomédicas (CEIB) "Dr. Uriel Guevara Guerrero" de la Universidad Nacional Autónoma de Nicaragua (UNAN) León.

\section{RESULTADOS}

\section{Perfil socio-demográfico de los participantes}

En su mayoría, $81 \%$ de los participantes fueron hombres; la edad promedio de la población de estudio fue de 34 años, con la edad mínima 18 años y máxima 67 años. Todos los participantes fueron nicaragüenses procedentes de 14 departamentos del país, 59\% de la capital, Managua. El 96\% se identificaron dentro del grupo étnico o raza mestiza (mixto) y el $4 \%$ afro-descendientes.

La mayoría, el 60\% declararon vivir en la casa de su familia de origen, antes de ingresar al Centro. De un total de los participantes el 65\% expresaron no tener pareja y el 55\% no tenían ingresos económicos propios. El 40\% de los parti- cipantes alcanzaron un nivel medio de educación (secundaria completa e incompleta), el 38\% nivel básico (primaria completa e incompleta), el 18\% educación superior y el 5\% manifestaron ser analfabetas (no saben leer, ni escribir).

\section{Características relacionadas con el consumo de sustancias psicoactivas}

La tabla 1 muestra que el alcohol con el 71\%, fue identificado como la principal sustancia por la que los participantes reciben atención actualmente. Entre otras principales sustancias, marihuana figuró con el 11.5\% de consumo, el 26\% como segunda sustancia de importantica y con el 15\% tercera sustancia. El 12\% de los participantes reflejó el consumo de crack como principal sustancia de tratamiento, el $28.5 \%$ como segunda sustancia de importancia y el $20 \%$ tercera sustancia. El consumo de cocaína también fue presente en tres momentos: como principal sustancia de tratamiento con el $5.4 \%$, segunda sustancia con el $28.5 \%$ y tercera con el $8.5 \%$. Las sustancias inhalables figuraron como una tercera droga de importancia para el tratamiento con el 3.1\% de consumidores.

Tabla 1 - Características relacionadas con el consumo de sustancias psicoactivas de los pacientes atendidos en centros de tratamiento de abuso/dependencia de drogas ManaguaNicaragua, $2010(n=130)$

\begin{tabular}{llrr}
\hline Variable & Categoria & \multicolumn{1}{c}{ n } & \multicolumn{1}{c}{$\%$} \\
\hline \multirow{4}{*}{ Principal sustancia } & Alcohol & 92 & 71.0 \\
& Marihuana & 15 & 11.5 \\
& Crack & 16 & 12.3 \\
& Cocaína & 7 & 5.4 \\
& Alcohol & 11 & 8.5 \\
Segunda sustancia & Marihuana & 34 & 26.2 \\
& Crack & 37 & 28.5 \\
& Cocaína & 10 & 7.7 \\
& Ninguna & 38 & 29.2 \\
& Alcohol & 9 & 6.9 \\
Tercera sustancia & Marihuana & 20 & 15.4 \\
& Crack & 26 & 20.0 \\
& Cocaína & 11 & 8.5 \\
& Inhalantes & 4 & 3.1 \\
& Ninguna & 60 & 46.1 \\
\hline
\end{tabular}

La droga más consumida durante los últimos 30 días, antes de ingresar al Centro, fue el alcohol con el $90 \%$ de consumo, seguido por el crack, con el $66 \%$, el $51 \%$ de ganja (preparado de marihuana) y el 
$37 \%$ de cocaína. En menor porcentaje el consumo fue distribuido entre otras 12 drogas, tales como inhalantes con el $13 \%$ de consumo, el $12 \%$ otros preparados de marihuana, el $8 \%$ pasta de coca, el 5\% abuso de medicamentos prescritos por médicos y de benzodiacepinas, el $5 \%$ anfetaminas y otros preparados de cocaína, el 4\% heroína y metanfetaminas, el3\% LSD, el $0.8 \%$ barbitúricos y esteroides anabólicos.

Con relación a la edad de inicio de consumo de sustancias psicoactivas, el mayor porcentaje se concentró en el rango de "13 a 17 años", con el 59\% de consumo de una primera droga, con el $51 \%$ de alcohol y el $42 \%$ de policonsumo de sustancias. La edad promedio de iniciar una primera droga fue de 17 años, con la edad mínima ocho años y máxima 54 años.

El $42 \%$ de los participantes manifestaron nunca recibir algún tratamiento por consumo de drogas antes de ingresar al Centro, 20\% una vez, el 33\% algunas veces y el 5\% muchas veces.

\section{Antecedentes y situación actual de los problemas de salud mental}

En su mayoría, el 59\% de los pacientes, notificaron haber tenido diagnósticos previos de algunos problemas de salud mental, entre éstos el $31 \%$ de ansiedad, el 32\% de depresión, el 11\% de trastorno bipolar y el $4 \%$ de esquizofrenia. El 38\% de los pacientes manifestaron recibir actualmente el tratamiento por ansiedad, el 24\% de depresión, el $9 \%$ de trastorno bipolar y $3 \%$ de esquizofrenia. El 22\% de los pacientes declararon estar recibiendo el tratamiento en el mismo Centro de atención por abuso/dependencia de drogas con una duración de "menos de un año".

\section{Presencia de distress psicológico}

Con relación a los valores de las puntuaciones de la escala K-10 de distress psicológico, éstos variaron entre 10 y 43 (promedio=19; mediana=17). La prevalencia del distress psicológico a nivel "severo" y "muy severo" fue de 35\%, el nivel "moderado" de $23 \%$ y "leve" $42 \%$.

Con relación a las variables sexo y el distress psicológico, el gráfico 1 muestra que las mujeres, en comparación con los hombres, presentaron mayores porcentajes en el distress moderado, severo y muy severo y menor porcentaje en el distress leve.

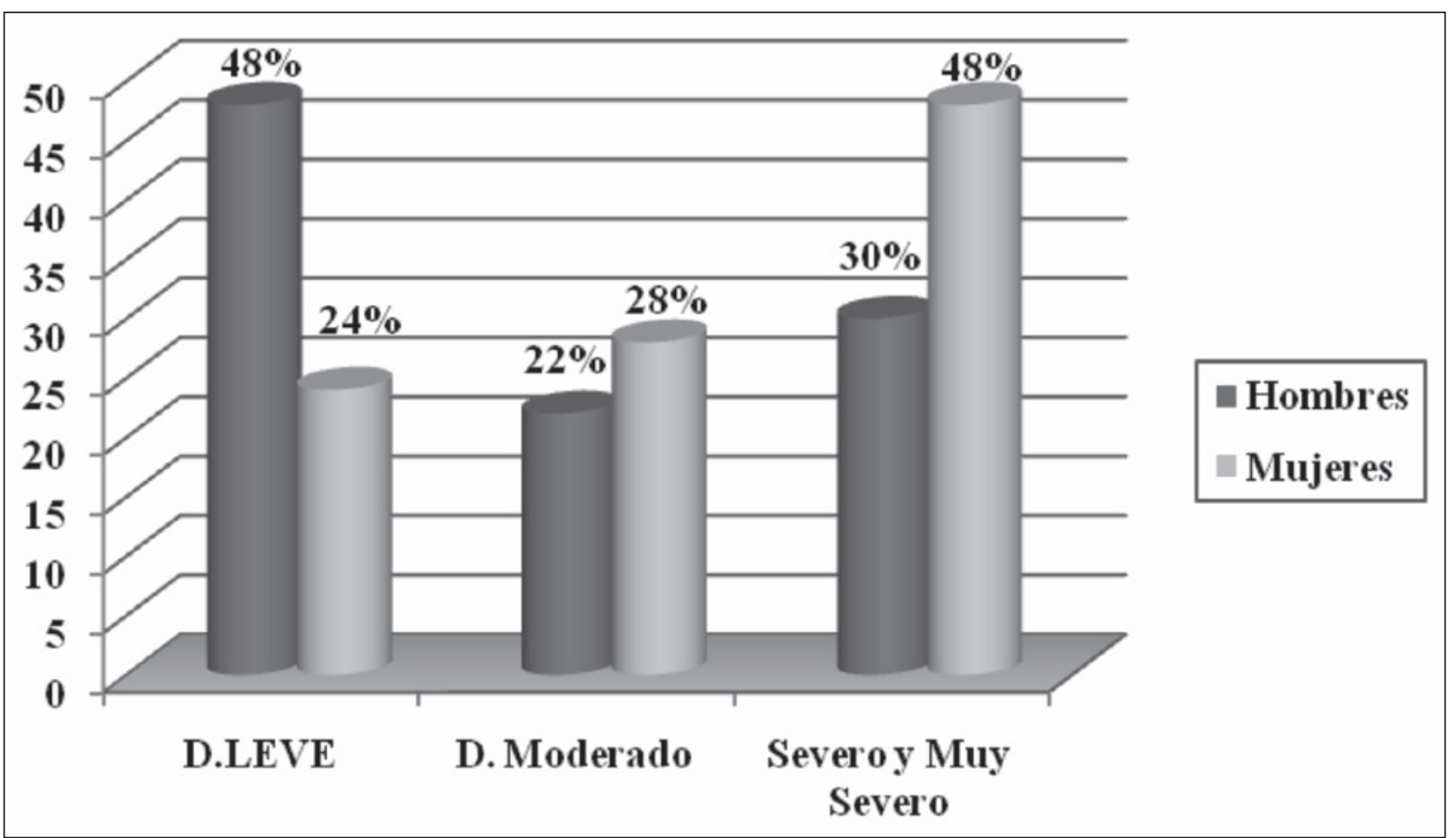

Gráfico 1 - Contingencia del porcentaje del comportamiento del distress entre los hombres y mujeres atendidos en centros de tratamiento de abuso/dependencia de drogas Managua-Nicaragua, 2010 (n=130)

Con relación a la edad cronológica, el gráfico 2 muestra que en su mayoría el $62 \%$, de los pacientes de 40 años en adelante, manifestaron un nivel leve del distress y el $42 \%$, de los pacientes con niveles del distress severo y muy severo se ubicaron entre 18 y 23 años de edad. 


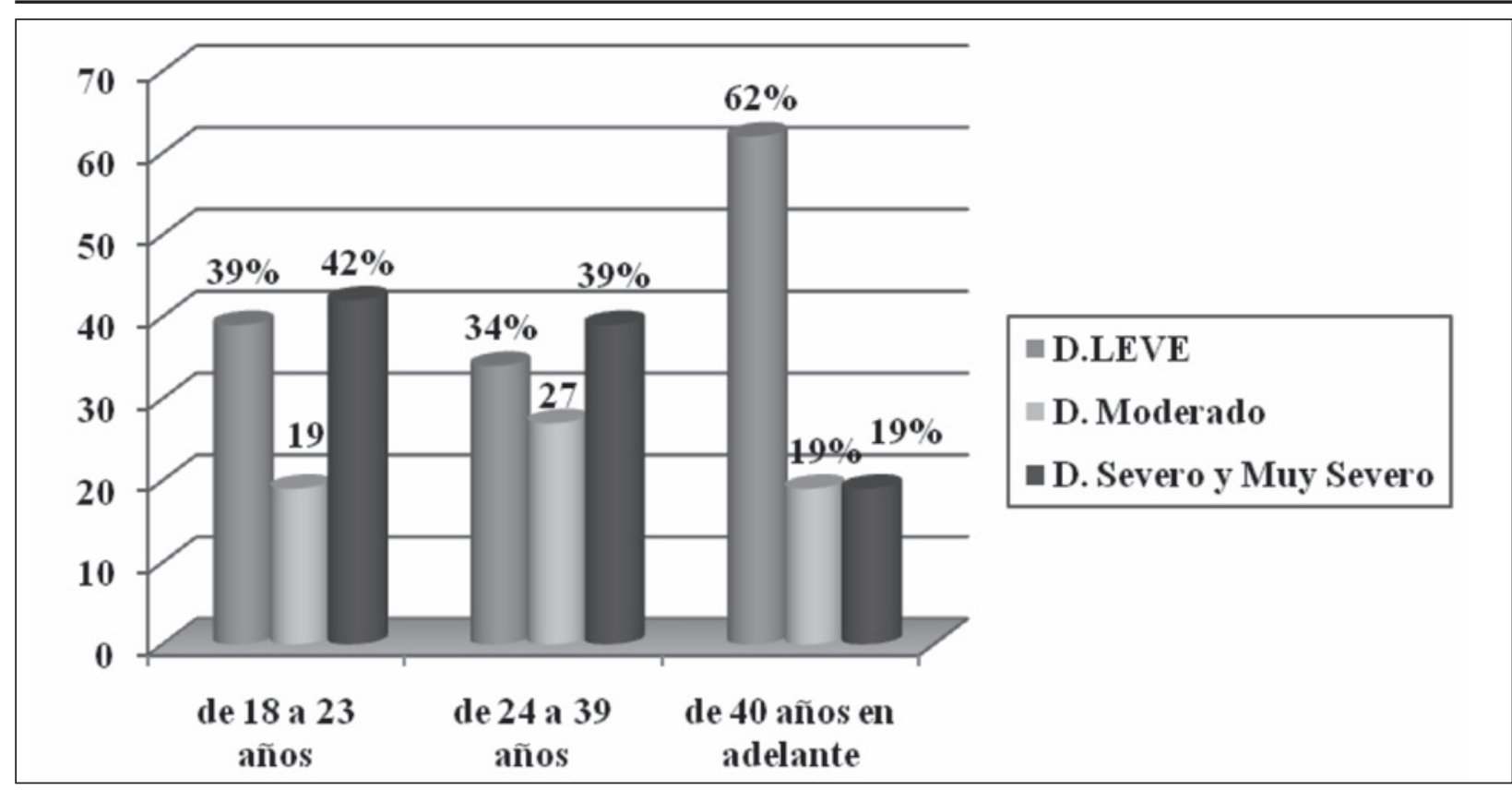

Gráfico2 - Contingencia del porcentaje del comportamiento del distress entre los rangos de edad cronológica de los pacientes atendidos en centros de tratamiento de abuso/dependencia de drogas Managua-Nicaragua, $2010(n=130)$

El gráfico 3 muestra la contingencia del comportamiento del distress en pacientes que han tenido un diagnóstico previo de algún problema de salud mental y los que no lo han tenido, en ambos casos, presentaron un comportamiento casi similar: el $36 \%$ de los pacientes que fueron diag- nosticados y el $33 \%$ que no fueron diagnosticados presentaron el distress severo y muy severo, el $24 \%$ de los pacientes que fueron diagnosticados y el $23 \%$ que no fueron diagnosticados presentaron el distress moderado.

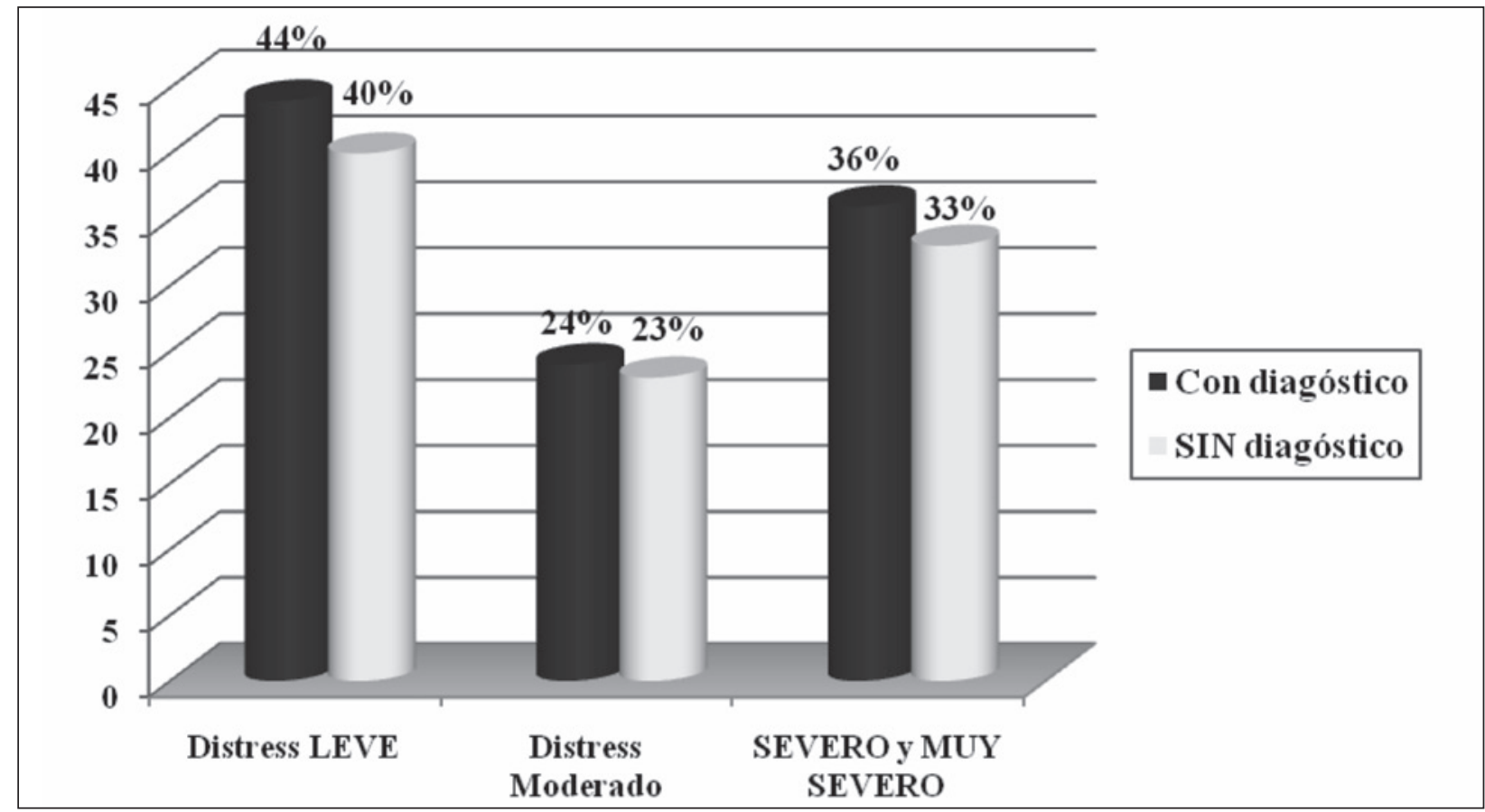

Gráfico 3 - Contingencia del porcentaje del comportamiento del distress en pacientes que han tenido o no un diagnóstico previo de salud mental. Managua-Nicaragua, 2010 (n=130) 
El gráfico 4 muestra la contingencia del comportamiento de distress en pacientes que están siendo tratados actualmente por diferentes problemas de salud mental; la mayoría manifestaron experimentar síntomas del distress con un alto porcentaje: $47 \%$ en tratamiento por ansiedad, $48 \%$ en tratamiento por depresión, $67 \%$ en tratamiento por trastorno bipolar y todos los pacientes tratados por esquizofrenia.

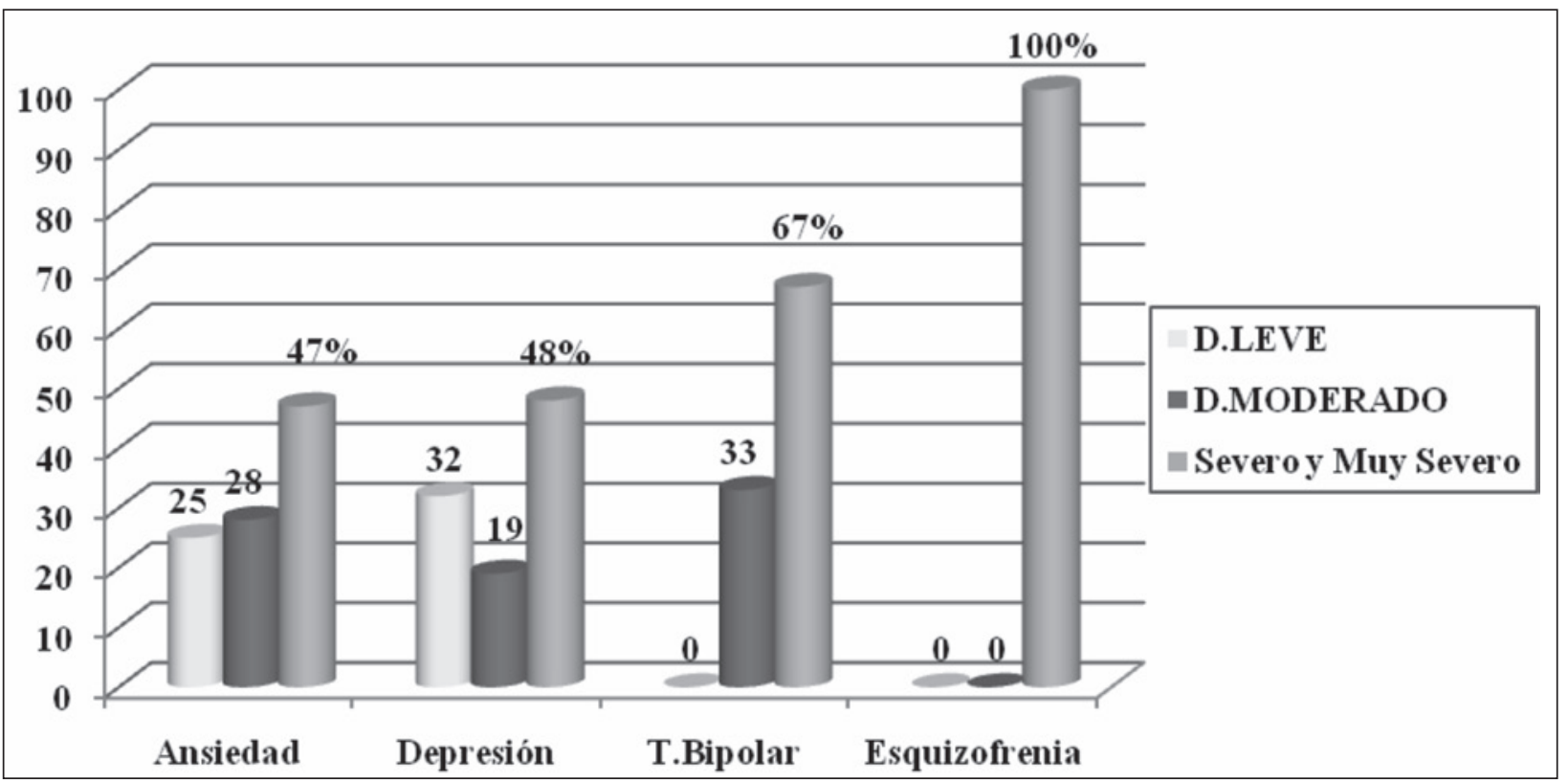

Gráfico 4 - Contingencia del porcentaje del comportamiento del distress en pacientes que están siendo tratados actualmente por problemas de salud mental. Managua-Nicaragua, $2010(n=46)$

\section{DISCUSIÓN}

En la literatura científica, entre diferentes autores, se puede encontrar un determinado nivel de coincidencia para algunas variables que describen el perfil de los pacientes que acuden a centros de tratamiento por drogodependencia. El perfil socio-demográfico de la población de este estudio es semejante al encontrado por la mayoría de los autores consultados..$^{12-15}$ Se trata de varones, -el 81\%-, con una edad media de 34 años. En diferentes muestras de los estudios realizados en México, USA y Europa, predominan hombres, con un promedio de $80 \%$, en torno a los 30 años de edad, ${ }^{15-18}$ el porcentaje de sujetos solteros o sin pareja oscila entre $60 \%$ y $68 \%$, que también coincide con la situación conyugal de los sujetos de este estudio, el 65\% no tienen pareja. Otra característica de coincidencia es el $60 \%$ de los sujetos estudiados vivieron en domicilio familiar antes de ingresar al Centro; en otros estudios este porcentaje oscila entre el $60 \%$ a $80 \% .12,14$

Otro dato interesante es con relación a los diagnósticos previos de la población estudiada, el $59 \%$ de los pacientes han tenido diagnósticos previos de algunos problemas de salud mental.
Los mismos datos se pueden encontrar en diferentes estudios. ${ }^{19-20}$ Entre el 31-65\% de los individuos que abusaron de sustancias psicoactivas alguna vez en su vida, también tuvieron una historia de al menos un desorden mental, y cerca de $51 \%$ de los mismos, con uno o más desordenes de salud mental a lo largo de su vida, tenia al menos una historia de trastornos por abuso de sustancias. ${ }^{21} \mathrm{Y}$, por otro lado, la tasa de comorbilidad es elevada en los Centros de tratamiento para el abuso de drogas, porque las personas que tienen ambos problemas buscan el tratamiento más frecuentemente que aquellos que sólo tienen un problema. ${ }^{5}$

En el presente estudio la prevalencia del distress psicológico fue el 35\%. Entre los aspectos epidemiológicos de la comorbilidad descritos en la literatura no parece existir unanimidad ya que las investigaciones aportan datos muy diferentes. ${ }^{22}$ Estas divergencias se relacionan, probablemente, con distintos problemas metodológicos, como el tipo de instrumento empleado, ${ }^{23}$ la selección de la muestra, ${ }^{24} \mathrm{y}$, sobre todo, el tiempo transcurrido desde el inicio de la abstinencia. ${ }^{23}$ Esta variabilidad de resultados se observa también con respecto al tipo concreto de psicopatología que concurre en 
el adicto. ${ }^{21,25}$ Los trastornos más frecuentes en los adictos parecen ser los trastornos de ansiedad y de estado de ánimo,; ${ }^{27}$ la prevalencia de trastornos de ansiedad oscila entre el $55 \%{ }^{20}$ y el $65 \%{ }^{26}$ y en el caso de los trastornos del estado de ánimo, los datos se sitúan entre el $24 \%{ }^{26}$ y el $33 \% .{ }^{27}$ Debido a que el valor del distress psicológico severo y muy severo sugiere una alta probabilidad de tener un trastorno de ansiedad o depresión, la prevalencia encontrada en este estudio demuestra que los pacientes de los centros de tratamiento en Nicaragua comparten una realidad clínica similar de los usuarios en otros países. Esto una vez más subraya la importancia que tiene la identificación del trastorno primario y la intervención oportuna, determinando necesidades terapéuticas sobre los efectos psicopatológicos generados, sean éstos por el abuso de drogas o cuadros clínicos de los trastornos mentales.

Con relación a las variables sexo y el distress psicológico, se observó que los pacientes con un distress moderado, severo y muy severo fueron mayormente mujeres que hombres. Varios estudios comparativos de hombres y mujeres en tratamiento de drogas han reflejado un comportamiento similar; las mujeres presentaban mayores tasas de depresión, trastorno de estrés postraumático y otros trastornos de ansiedad, mientras que en hombres lo común fue el trastorno de personalidad antisocial. ${ }^{28}$

Un hallazgo importante es con relación a los pacientes que están siendo tratados actualmente por problemas de salud mental, ellos manifestaron experimentar síntomas del distress con un porcentaje elevado. Una de las posibles explicaciones de esta situación podría hallarse en el déficit de los sistemas asistenciales. La ausencia de cuidados integrados de comorbilidad en estos Centros pudo haber generado dificultades para responder a las demandas del entorno, y en un futuro pondría en peligro la recuperación y la estabilidad de estos pacientes.

\section{CONCLUSIONES}

La prevalencia del 34\% de la comorbilidad encontrada en la población de estudio y el hecho de que estos pacientes persisten en experimentar síntomas del distress psicológico sin importar si han tenido o no un diagnóstico previo de problemas de salud mental o estar tratados actualmente por estos problemas, incrementa el valor de las necesidades asistenciales emergentes de esta po- blación, y sugiere una revisión sistemática de las políticas y estrategias de intervención y el fortalecimiento de ambos sistemas involucrados, Sistema de Salud y de Drogodependencia.

\section{Limitaciones}

El difícil acceso a los centros de atención por drogodependencia y sus condiciones de ser organizaciones no gubernamentales de tipo alberges o refugios carentes de profesionales especializados, en su gran mayoría, presenta una limitación para poder generalizar los resultados sobre la situación clínica que tienen los pacientes de otros centros del país. El tipo de diseño transversal de este estudio presenta otra limitación, porque no permite conocer la dinámica de los eventos relacionados con la patología dual de los pacientes.

\section{Recomendaciones}

Mejorar la asistencia de los pacientes drogodependientes, tomando conciencia de la magnitud y especificidad de la problemática.

Proporcionar formación de recursos humanos que atienden a los pacientes en centros de tratamiento sobre patología dual.

Desarrollar una mejor coordinación entre los servicios de salud mental y drogodependencia, así como mejorar la legislación del país sobre el tema.

Fortalecer el liderazgo del Consejo Nacional de Lucha contra las Drogas y el Ministerio de Salud de Nicaragua con relación a la atención de la población con problemas de drogodependencia.

\section{AGRADECIMIENTOS}

Esta investigación se llevó a cabo gracias al apoyo financiero del Gobierno de Canadá, la Comisión Interamericana para el Control del Abuso de Drogas (CICAD) de la OEA y al Centro de Adicciones y Salud Mental (CAMH) de Canadá y también no hubiera sido posible sin apoyo del claustro de profesores del CAMH que con su agudeza docencia guiaron al grupo CICAD-IV en este proyecto multicéntrico. Agradezco el apoyo de la dirección del Instituto Nacional Contra Alcoholismo y Drogodependencia (ICAD) de Managua y a los directores de los Centros de tratamiento, especialmente REJESAD por apoyar con entusiasmo la investigación y considerarla como una oportunidad para conocer y mejorar la realidad de sus usuarios. 


\section{REFERENCIAS}

1. Hersh DF, Modesto-Lowe V. Drug abuse and mood disorders.In: Henry BK, Bruce J, Rounsaville MD, editors. Dual diagnosis and treatment. New York (US); Marcel Dekker, Inc.; 1998. p. 177-201.

2. Kranzler HR, Mason B, Modesto-Lowe V. Prevalence, diagnosis, and treatment of comorbid mood disorders and alcoholism. In: Kranzler HR, Rounsaville B, editors. Dual diagnosis and treatment. New York (US): Marcel Dekker, Inc. 1998. p. 107-36.

3. Skinner WJW, editor. Treating concurrent disorders a guide for counselors. Toronto (CA): Centre for Addiction and Mental Health; 2005.

4. Hasin DS, Grant BF. The co-occurrence of DSMIV alcohol abuse in DSM-IV alcohol dependence: results of the National Epidemiologic Survey on Alcohol and Related Conditions on heterogeneity that differ by population subgroupArch Gen Psychiatry. 2004 Sep;61(9):891-6.

5. Grant BF. The influence of comorbid major depression and substance use disorders on alcohol and drug treatment: results of a national survey. In: National Institute on Drug Abuse (NIDA). Treatment of drug-dependent individuals with comorbid mental disorders. Rockville (US): NIH; 1997.

6. Organización de los Estados Americanos, Comisión Interamericana para el Control del Abuso de Drogas. Nicaragua: Evaluación del Progreso de Control de Drogas, 2005-2006. Mecanismo de Evaluación Multilateral. Washington D.C. (US): OEA, CICAD; 2006.

7. Organización de los Estados Americanos, Comisión Interamericana para el Control del Abuso de Drogas. Brasil: Evaluación del Progreso de Control de Drogas, 2005-2006. Mecanismo de Evaluación Multilateral. Washington D.C. (US): OEA, CICAD; 2006.

8. Comisión Interamericana para el Control del Abuso de Drogas. Informe comparativo 7 países, encuestas escolares a nivel nacional: El Salvador, Guatemala, Nicaragua, Panamá, Paraguay, República Dominicana y Uruguay. OEA/CICAD/ OID/SIDUC; 2004.

9. Fletes C. Informe de Ingresos por alcoholismo y drogas, 1997-2005. Programa Nacional de Salud Mental. Dirección General de Servicios de Salud Ministerio de Salud República de Nicaragua, 2006.

10. Zimmerman M. To screen or not to screen: conceptual issues in screening for psychiatric disorders in psychiatric patients with a focus on the performance of the psychiatric diagnostic screening. Int J Ment Health Addiction. 2008 Jan; 6(1):53-63.

11. Andrews G, Slade T. Interpreting scores on the Kessler Psychological Distress Scale (K10). Aust N Z J Public Health. 2001 Dec; 25(6):494-7.
12. Kang SY, Delon G. Correlates of drug injection behaviors among methadone outpatients. Am J Drug Alcohol Abuse 1993; 19:107-18.

13. Torrens M, San L, Martínez A, Castillo C, DomingoSalvany A, Alonso J. Use of the Nottingham Health Profile for measuring health status of patients in methadone maintenance treatment. Addiction. 1992 Jun; 92:707-16.

14. Puigdollers E, Domingo-Salvany A, Brugal MT, Torrens M, Alvaros J, Castillo C, et al. Characteristics of heroin addicts entering methadone maintenance treatment: quality of life and gender. Subst Use Misuse. 2004 Jul; 39:1353-68.

15. Cacciola JS, Alterman AI, Rutherford MJ, McKay JR, McLellan AT. The early course of change in methadone maintenance. Addiction. 1998 Jan; 93(1):41-9.

16. Lee SS, Hollinrake JB, Ng MH. Changing behavioural pattern of drug users in Hong-Kong 1991-1995. Addiction. 1998 Apr; 93(4):541-8.

17. Domingo-Salvany A, Perez K, Torrens M, Bravo MJ, Anto JM, Alonso J. Methadone treatment in Spain, 1994. Drug Alcohol Depend. 1999 Aug 2; 56(1):61-6.

18. Egea Zapata C, Carceles M, Osuna Carrillo De Albornoz E, Romero Mf, Maldonado AL. Perfil de los usuarios de una unidad móvil de drogodependencias en el sureste de España. Addiction. 2005; 17(4):307-14.

19. Mari JJ, Jorge MR, Kohn R. Epidemiologia dos transtornos psiquiátricos em adultos. In: Mello MF, Mello AAF, Kohn R, organizadores. Epidemiologia da saúde mental no Brasil. Porto Alegre (RS): Artmed; 2007. p.119-41.

20. Colin C. Substance abuse issues and public policy in Canada. Canada's federal drug Strategy. 2006 [acesso 2009 Jun 25]. Disponível em: www.parl. gc.ca/information/library/PRBpubs//prb0615-e. html

21. Driessen M, Meier S, Hil, A, Wetterling T, Lange W, Junghanns K. The course of anxiety, depression and drinking behaviours after completed detoxification in alcoholics with and without comorbid anxiety and depressive disorders. Alcohol Alcohol. 2001 May-Jun; 36(3):249-55.

22. Weiss RD, Mirin SM, Griffin ML. Methodological considerations in the diagnosis of coexisting psychiatric disorders in substance abusers. Br J Addict. 1992 Feb; 87(2):179-87.

23. Valbuena A, Largo R, Quintero J, García E, Correas J. Comorbilidad en alcohólicos ingresados. Implicaciones clínicas y sociosanitarias. Adicciones, 2001; 13, 297-304.

24. Schuckit MA, Tipp JE, Bergman M, Reich W, Hesselbrock VM, Smith TL. Comparison of induced and independent major depressive disorders in 2945 alcoholics. Am J Psychiatry. 1997 Jul; 154(7):948-57. 
25. Tomasson K, Vaglum P. A nationwide representative sample of treatment-seeking alcoholics: a study of psychiatric comorbidity. Acta Psychiatr Scand. 1995 Nov; 92(5):378-85.

26. Casas M, Guardia J. Patología psiquiátrica asociada al alcoholismo. Adicciones, 2002: 14:195-219.

27. Schneider U, Altman A, Baumann M, Bernzen J, Bertz B, Bimber U. et al. Comorbid anxiety and affective disorder in alcohol-dependent patients seeking treatment: the first multicentre study in Germany. Alcohol Alcohol. 2001 May-Jun; 36(3):219-23.

28. Brady K, Myrick H, Sonne S. Comorbid addiction and affective disorders. En: Graham A, Schultz T, editors. Principles of addiction medicine. Mayland (US): American Society of Addiction Medicine, Maryland, USA; 1998. p. 983-92. 